\title{
Impact of miR-208 and its Target Gene Nemo-Like Kinase on the Protective Effect of Ginsenoside Rb1 in Hypoxia/Ischemia Injuried Cardiomyocytes
}

\author{
Xu Yan ${ }^{a, b}$ Jianxun Liuc Hongjin Wu ${ }^{a}$ Yuna Liu ${ }^{d}$ Sidao Zheng ${ }^{d}$ \\ Chengying Zhang ${ }^{d}$ Cui Yang ${ }^{d}$
}

aBeijing Haidian Hospital, Haidian Section of Peking University Third Hospital, 29 Zhongguancun Dajie, Beijing, 'bepartment of Pathophysiology, Beijing Neurosurgical Institute / Beijing Tiantan Hospital, Capital Medical University, Beijing, Institute of Basic Medical Sciences of Xiyuan Hospital, China Academy of Chinese Medical Sciences, Beijing, ${ }^{\mathrm{d} B e i j i n g ~ H o s p i t a l ~ o f ~ I n t e g r a t e d ~ T r a d i t i o n a l ~ C h i n e s e ~ a n d ~}$ Western Medicine, Beijing, China

\section{Key Words}

Cardiomyocytes • Hypoxia/ischemia • Ginsenoside Rb1 • miR-208 • Nemo-like kinase • Apoptosis

\begin{abstract}
Background/Aims: Ginsenoside Rb1 (GS-Rb1) is one of the most important active pharmacological extracts of the Traditional Chinese Medicine ginseng, with extensive evidence of its cardioprotective properties. Mir-208 has been shown to act as a biomarker of acute myocardial infarction in vivo studies including man. However the impact of miR-208 on the protective effect of GS-Rb1 in hypoxia/ischemia injured cardiomyocytes remains unclear. The current study aims to investigate the target gene of miR-208 and the impact on the protective effect of GS-Rb1 in hypoxia/ischemia (H/I) injuried cardiomyocytes. Materials and Methods: Primary cultures of neonatal rat cardiomyocytes (NRCMs) was subjected to the H/I conditions with or without GS-Rb1. Cell viability was calculated by MTT assay and confirmed by flow cytometry analysis. Mir-208 was then detected by qRT-PCR. Luciferase reporter assay was carried out to detect the target gene of Mir-208. Then the NRCMs were transfected with miR-208 mimics and inhibitors to evaluate the impact on cardioprotective properties of Rb1. Results: The miR-208 expression level was clearly upregulated in the H/I treated NRCMs accompanied by the percentage of the apoptotic cells which could be reversed by GS-Rb1 pretreatment. The nemo-like kinase (NLK) mRNA and protein expression levels were decreased in H/I group measured by RT-PCR and western blotting. Luciferase activity assay was then carried out to identify that NLK may be a direct target of mir-208. MTT assay showed that miR-208 inhibitor slightly decreased the protective effect of Rb1 on the $\mathrm{H} / \mathrm{I}$ impaired
\end{abstract} $X$. Yan and J. Liu contributed equally to this work.

Hongjin Wu

KARGER
Beijing Haidian Hospital, 29 Zhongguancun Dajie, Haidian District, 
NRCMs. However, results showed no statistical difference. Conclusions: These findings proved that NLK was a direct target of mir-208 and miR-208 act indirectly during Rb1 protecting $\mathrm{H} / \mathrm{I}$ impaired NRCMs and further researches were needed to explore the relationship that microRNAs and other signal pathways in the protective effect of GS-Rb1 on the hypoxia/ ischemia injuries in cardiomyocytes.

(C) 2016 The Author(s)

Published by S. Karger AG, Basel

\section{Introduction}

Myocardial infarction (MI), the one of the leading causes of death throughout the world, presents a major challenge to the health services because cases contribute significantly to healthcare costs and the degree of disability among the adult population. There is now evidence that microRNAs (miRNAs) are involved in the causes, processes, development and consequences of MI, making it possible that they can act a target for the drug design [1-4].

Nemo-like kinase (NLK), a Wnt/ $\beta$-catenin signaling inhibitor, regulates diverse signaling processes by phosphorylating several transcription factors [5]. Activation of Wnt/ $\beta$-catenin signaling pathway could directly induce apoptosis in many mammalian cells. In cardiomyocytes, Wnt/ $\beta$-catenin signaling has a multifunctional role in heart cell generation, proliferation and differentiation [6,7]. Further evidence suggests that Wnt/ $\beta$-catenin signaling is involved in heart disease as well as myocardial infarction [8-11].

MicroRNAs (miRNAs) have emerged as a novel class of endogenous, small $(<24$ nucleotide), noncoding RNAs with strong biological functions in the cardiovascular system $[12,13]$. Mature miRs regulate the repression of specific target gene translation and/or promote the degradation of their transcribed mRNAs by binding to the 3'-untranslated regions (UTR) of the target genes $[14,15]$. miRNAs provide an additional post-transcriptional layer of spatial and temporal control of developmental and homeostatic events by altering levels of critical regulators within complex genetic pathways [16-18]. As a group, it is estimated that miRNAs are estimated regulate $30 \%$ of the genes in the human genome [19]

Further evidence implicates miRNA function as integral in regulatory cellular functioning, including proliferation, apoptosis, and many physiological pathways. MiR-208 is specifically expressed in the mammalian cardiomyocytes, being encoded by an intron of the $\alpha$-cardiac muscle myosin heavy chain gene (Myh6) and required for cardiomyocyte hypertrophy and fibrosis [20]. miR-208 sequences in humans, mice, rats, and dogs are identical, with premiRNA being highly conserved in mammals. In acute myocardial infarction in patients and animals, mir-208 is markedly increased in plasma [21-23] and even in urine [24], indicating that it could be a useful diagnostic biomarkers. However, the involvement of mir-208 in hypoxic- and ischemic-induced cardiomyocyte apoptosis is unclear.

The root of Panax ginseng is one of the most popular herbal medicines for the treatment of many ailments, particularly those associated with aging and cardiac deterioration, which has been used for several thousand years in China [25, 26]. Ginsenoside Rb1, one type of protopanaxadiol saponin, is one of the most important active compounds of ginseng [27], and an increasing amount of evidence has indicated that $\mathrm{Rb} 1$ possesses cardioprotective effects both in vitro and in vivo [28]. However, the target miRNA of Rb1 and the related mechanism has not been fully characterized. In the present study, we found that miR-208 expression was upregulated in the cardiomyocytes in vitro and accompanied with cell apoptosis induced by hypoxia/ischemia treatment, which could be ameliorated by Rb1 pretreatment. Additionally, the target gene of miR-208 was identified by luciferase reporter assay. Then, miR-208 mimics and miR-208 inhibitor was transfected to the neonatal rat cardiomyocytes to investigate the impact on the cardioprotective effect of Rb1 on the cardiomyocytes impaired by hypoxia/ ischemia treatment. 


\section{Cellular Physiology Cell Physiol Biochem 2016;39:1187-1195

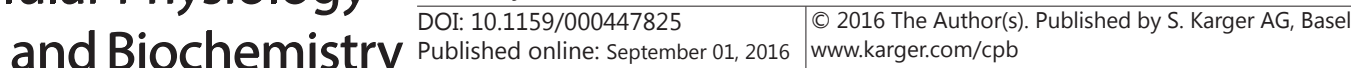 \\ Yan et al.: miR-208 Targets Nemo-Like Kinase in Cardiomycytes}

\section{Materials and Methods}

Materials

GS-Rb1 (catalog number \#110704), purchased from National Institutes for food and drug Control, was dissolved in phosphate-buffered saline (PBS) to create a stock solution for subsequent dilution. Dulbecco's Modified Eagle Medium (DMEM) and fetal bovine serum (FBS) were obtained from Gibco (Grand Island, NY, USA). DMSO and 3-(4, 5-dimethyl-thiazol-2-yl)-2,5-diphenyltetrazoliumbromide (MTT) were obtained from Sigma (St. Louis, MO, USA). Annexin V-FITC/PI kit was purchased from Kaiji Biological Engineering Institute. Cell Lysis Buffer for Western blotting, and IP and Enhanced BCA Protein Assay Kit were obtained from Beyotime (Haimen, China). The primary antibody against NLK (catalog number \# Ab97642) was purchased from Abcam. Horseradish peroxidase (HRP)-conjugated anti-rabbit IgG secondary antibody (catalog number \# A0545) was obtained from Sigma. Enhanced chemiluminescence kit was obtained from Millipore (Billerica, MA, USA). miRNA extracting kit (CW0627) was purchased from Beijing Cowin Biotech Co., Ltd.

Culture of neonatal rat cardiomyocytes and transfection (NRCMs)

Primary cultures of NRCMs from 12-24 h old Sprague Dawley rats (Vital River Laboratories, Beijing, China) was prepared by means of gentle serial trypsinization as previously described [29]. All experiments were approved by the Beijing Ethics Committee for the Use of Experimental Animals. The NRCMs were plated in collagen-coated 96 - or 6-well plates and maintained at $37^{\circ} \mathrm{C}$ in a $5 \% \mathrm{CO}_{2} / 95 \%$ air humidified incubator in DMEM containing 10\% (v/v) fetal bovine serum, $100 \mathrm{U} / \mathrm{mL}$ penicillin and $100 \mathrm{mg} / \mathrm{mL}$ streptomycin. The following experiments used spontaneously beating cardiomyocytes 48-72 h after plating.

The miRNA mimics and inhibitors were synthesized by Hanbio Biotechnology Co.Ltd. (Shanghai, China) and were transfected into cells using Lipofect-amine 2000 (Invitrogen, Carlsbad, CA, USA). Total RNA and protein were prepared at $48 \mathrm{~h}$ after transfection for qPCR and Western blot analysis, respectively.

\section{Hypoxia/ischemia treatment}

For the H/I protocol, hypoxia was induced with the MGC AnaeroPack System in a AnaeroPack jar (Mitsubishi Gas Chemical Co., Tokyo, Japan), depleting the concentration of $\mathrm{O}_{2}$ to $10 \%$ in $2 \mathrm{~h}$. Ischemia was reached by replacing culture medium with DMEM without glucose (Gibco, Grand Island, USA) and serum. NRCMs with or without GS-Rb1 were placed in the AnaeroPack jar and then put into a $37^{\circ} \mathrm{C}$ incubator for 24 h. Control plates were kept under normoxic conditions for the corresponding time.

MTT assay

NRCMs viability was determined by the MTT assay. Cardiomyocytes were plated on 96-well dishes at $2 \times 10^{4}$ cells/well. MTT (5 mg/mL) was added to each well immediately after hypoxia/ ischemia treatment. Plates were incubated for $4 \mathrm{~h}$ at $37^{\circ} \mathrm{C}$, the medium aspirated from each well and $100 \mu \mathrm{L}$ of DMSO added to dissolve the formazan crystals. The optical density of each well was read at $492 \mathrm{~nm}$ using a Microplate Reader (Bio-Rad, Hercules, CA). Results are given as percentages of the control group (100\%).

\section{Apoptosis assay by Annexin V/PI staining}

NRCMs were harvested and washed with PBS at the indicated time-points. The percentage of normal (non-apoptotic) and apoptotic cells was measured by double supravital staining with Annexin-V and PI, using an Annexin V-FITC Apoptosis Detection kit (KeyGen, Nanjing, China). Flow cytometric analysis used a Cytomics FC500 flow cytometer with CXP software (Beckman Coulter, Fullerton, USA), the operator being blind of the group assignment.

\section{Western Blotting.}

NRCMs under different hypoxic and ischemic conditions were harvested and lysed with Cell Lysis Buffer for Western blotting and IP. Protein concentration was measured using a BCA Protein Assay Kit. Equal amounts of sample lysate were separated by sodium dodecyl sulfate polyacrylamide gel electrophoresis (SDS-PAGE) and transferred by electroblotting to a nitrocellulose membrane (Millipore). The membrane was blocked with 5\% non- fat milk in TBST buffer (20m M Tris-HCl, pH7.4, $150 \mathrm{mM} \mathrm{NaCl}$ and $0.1 \%$ Tween 20) overnight at $4^{\circ} \mathrm{C}$. The membrane was incubated with specific primary antibodies for $2 \mathrm{~h}$ and an IgG HRP conjugated specific secondary antibody for $1 \mathrm{~h}$ at room temperature. The signals were visualized

\section{KARGER}




\section{Cellular Physiology Cell Physiol Biochem 2016;39:1187-1195

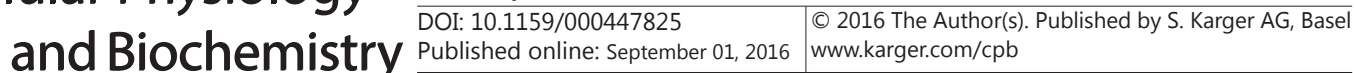 \\ Yan et al.: miR-208 Targets Nemo-Like Kinase in Cardiomycytes}

Table 1. Primer pairs used for qRT-PCR

\begin{tabular}{ll}
\hline \multicolumn{1}{c}{ Primers } & \multicolumn{1}{c}{ Sequences 5'-3' } \\
\hline Mir-208 RT primer & CTCAACTGGTGTCGTGGAGTCGGCAATTCAGTTGAGGCTTTTTG \\
Mir-208 Forward primer & ACACTCCAGCTGGGATAAGACGAGCA \\
Mir-208 Reverse pimer & TGGTGTCGTGGAGTCG \\
U6 Forward primer & CTCGCTTCGGCAGCACA \\
U6 Reverse primer & AACGCTTCACGAATTTGCGT \\
NLK Forward primer & CTAGATGAAGGGCGGCTGAG \\
NLK Reverse primer & GAGCGACGGTGGAACTGATA \\
GAPDHForward primer & CACCATCTTCCAGGAGCGAG \\
GAPDH Reverse primer & AAATGAGCCCCAGCCTTCTC \\
\hline
\end{tabular}

by enhanced chemiluminescence (ECL) (Pierce) on a Syngene G: BOX Chemi gel documentation system (Syngene, Cambridge, UK). Densitometric values were normalized in each group using GAPDH as an internal control.

RNA extraction and quantitative reverse transcription- polymerase chain reaction ( $q R T-P C R$ )

Rno-mir-208a (MIMAT0000880) mature sequence was: AUAAGACGAGCAAAAAGC. Total RNA from cells in control, $\mathrm{H} / \mathrm{I}$ treated, mir-208 mimic group or the negative control miRNA was isolated using the RNA extracting kit (Cowin Biotech) following the manufacturer's instructions.

Primer pairs used for qRT-PCR are given in Table 1. cDNA synthesis was done with $1 \mu \mathrm{g}$ total RNA using an RT-PCR reagent kit (TOYOBO), and cDNA quantified by PCR using the SYBR Green Master Mix (Roche). The PCR program was $95^{\circ} \mathrm{C}$ for 5 min followed by 40 cycles of $95^{\circ} \mathrm{C}$ for $20 \mathrm{~s}, 60^{\circ} \mathrm{C}$ for $30 \mathrm{~s}$, and $72^{\circ} \mathrm{C}$ for $20 \mathrm{~s}$. The relative expression level of NLK was normalized to that of GAPDH by the $2^{-\Delta \Delta C t}$ cycle threshold method. The relative fold-change in mir-208 expression level was calculated to that of U6 by the $2^{-\Delta \Delta c t}$ cycle threshold method.

Luciferase reporter assay

miR-208 target sequences were inserted between the XhoI and NotI restriction sites in the 3' UTR of the hRluc gene in the psiCHECK-2 vector (Promega, Madison, WI, USA). Fragments of NLK 3'UTR were amplified from the genomic DNA using the forward primer: 5'-ACACTCGAGAAGTTCAATGTGGAGACTTGAAAC-3' and the reverse primer: 5'-ATTAGCGGCCGCCATTGAAGTTAAAACTTCC-3'. Cells were cotransfected with $200 \mathrm{ng}$ psiCHECK2 constructs and $30 \mathrm{nM}$ miR-208 mimics or miR-negative control in 24-well plates. One day after transfection, firefly and Renilla luciferase activities were measured by the Dual-Glo Luciferase assay system (Promega). Normalized data were calculated as the quotient of Renilla/firefly luciferase activities. Three independent experiments were run in triplicate.

Construction of plasmids and transfection

For lentivirus-mediated overexpression of NLK, the NLK cDNA was inserted into a pLVX-Zs Green expression vector. Recombinant lentiviruses expressing NLK was produced by Genelive (Shanghai, China). The NRCMs cells were infected with concentrated virus according to the manufacturer's instructions. The NLK expression in the infected cells was validated by western blotting assay.

Statistical analysis

Data are given as means \pm SD, and Student's t-test was used for comparisons. $p<0.05$ was taken as a significant difference between groups.

\section{Results}

H/I Induced Cardiomyocytes apoptosis and cardioprotective effect of Rb1

Effect of $\mathrm{H} / \mathrm{I}$ induced NRCMs apoptosis was examined by the MTT assay and confirmed by Annexin V-FITC/propidium iodide (PI) double-staining followed by flow cytometry analysis (Fig. 1A). Compared with the control group, H/I treatment from 3 to $24 \mathrm{~h}$ decreased NRCMs 
viability from $77.7 \pm 4.2 \%$ to $25.7 \pm 3.2 \%$. With the presence of $\mathrm{Rb} 1(40 \mu \mathrm{M})$, cell viability increased for more than 2 times compared to the H/I 24 h group. To examine the effect of $\mathrm{H} / \mathrm{I}$ treatment on the cell death, flow cytometry analysis was performed and the results showed that the percentage of living cells after $24 \mathrm{~h} \mathrm{H} / \mathrm{I}$ treatment markedly decreased in $\mathrm{H} / \mathrm{I}$ group compared to the control group, and could be reduced by GS-Rb1 treatment (Fig. 1 B-D, B1 box).

\section{$N L K$ is a direct target of $m i R-208$}

The level of miR-208 was detected by real-time RT-PCR assay. The expression level of miR-208 in H/I group increased nearly 10 folds versus control, with the treatment of GS$\mathrm{Rb} 1$, the expression change of miR-208 could be reversed.

Since we have already shown that H/I treatment markedly unregulated mir-208 expression in NRCMs, to validate whether miR-208 regulates NLK directly through a putative binding site in NRCMs, we cloned NLK 3'-UTR in the predicted miRNA binding site into the luciferase gene (pSiCHECK2; Promega). Following cotransfection with the pSiCHECK2 vectors and miR-208 or the negative control miRNA, the upregulation of miR-208 in NRCMs transfected with miR-208 significantly decrease luciferase activity of the wild-type NLK 3'UTR (Fig. 2; P<0.05), clearly indicating that NLK is a direct target of miR-208.

\section{miR-208 negatively regulates NLK expression in NRCMS}

To investigate the correlation between miR-208 and NLK, miR-208 was upregulated in NRCMs by treatment with miR-208 for $48 \mathrm{~h}$. Expression of NLK mRNA and protein was measured by qRT-PCR and western blot analysis. NLK mRNA expression in cells transfected with the negative control vector was 6-fold higher than that in the cells transfected with miR-208. Western blot analysis showed that upregulated miR-208 leads to a corresponding decrease in endogenous NLK protein (Fig. 3), suggesting that miR-208 negatively regulates NLK expression.

Fig. 1. Effects of Rb1 on the hypoxica/ ischemia-induced NRCMs death. NRCMs with or without $\mathrm{Rb} 1$ were incubated with normal or $\mathrm{H} / \mathrm{I}$ conditions from $3 \mathrm{~h}$ to $24 \mathrm{~h}$. A. Cell viability was determined by MTT assay. B-D. NRCMs in control, $\mathrm{H} / \mathrm{I} 24 \mathrm{~h}$ and $\mathrm{H} / \mathrm{I}$ $24 \mathrm{~h}+\mathrm{Rb} 1$ group was stained with Annexin V-FITC/ PI and analyzed by flow cytometry. ** $\mathrm{p}<0.01,{ }^{* * *} \mathrm{p}<$ 0.001 versus control, \#\#p < 0.01 versus $\mathrm{H} / \mathrm{I} 24 \mathrm{~h}$ group. $(\mathrm{n}=3)$.

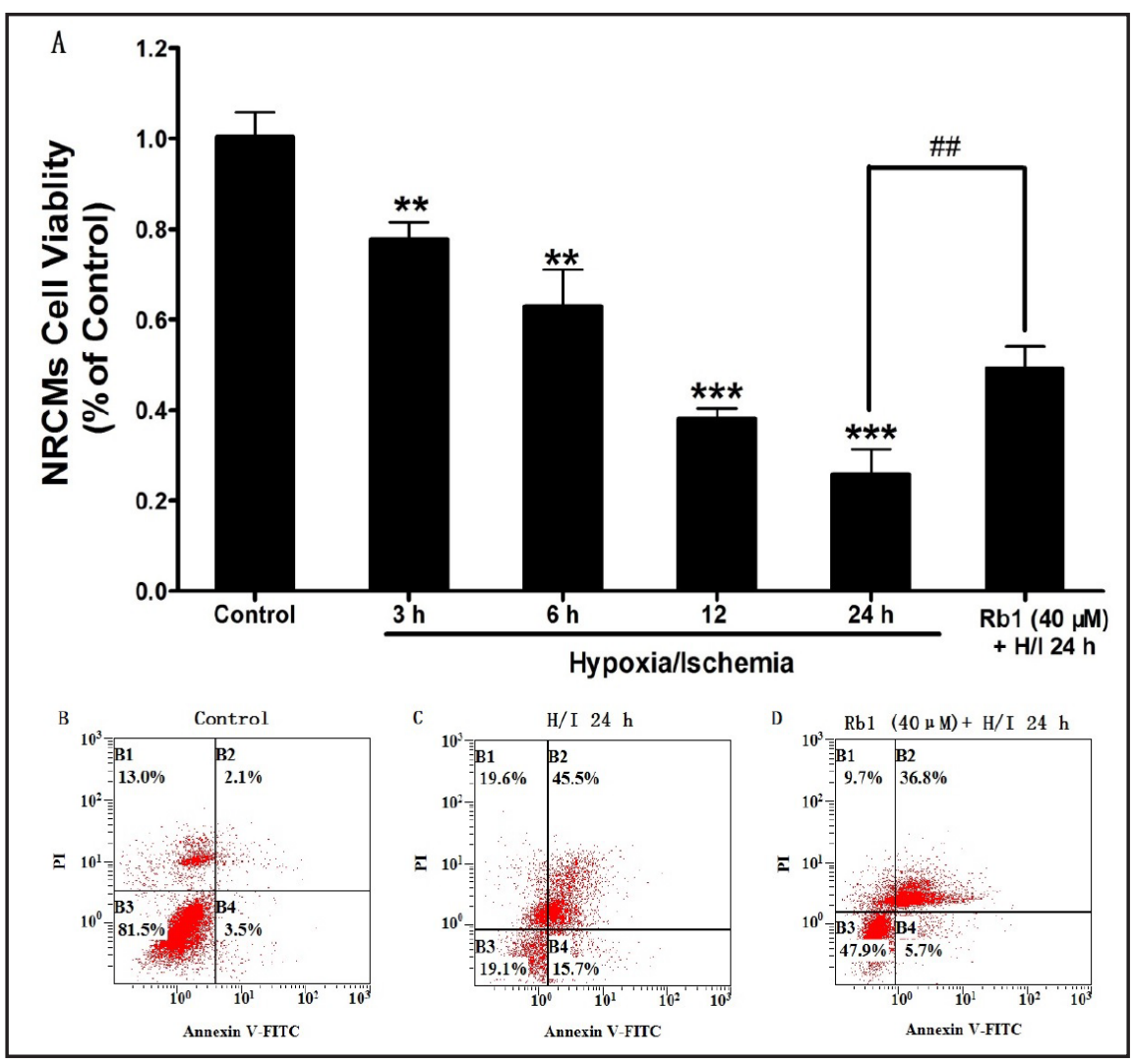


Fig. 2. Identification of target sites in the 3' UTR of rat NLK. A. qRT-PCR analysis of miR-208 in NRCMs. B. Construct map of psiCHECK-2/ NLK used for the luciferase assay. The 3'UTR of NLK was cloned into the vector after the luciferase gene to form a fusion transcript. C. Schematic diagram of miR-208 binding sites in the 3' UTR. D. Relative renilla luciferase activity of psiCHECK-2/ NLK in the presence of miR-208 or miR-neg expression vectors in NRCMs, and normalized to firefly luciferase activity. ${ }^{* * *} \mathrm{P}<0.01$ compared with those transfected with miR-neg expression vectors.

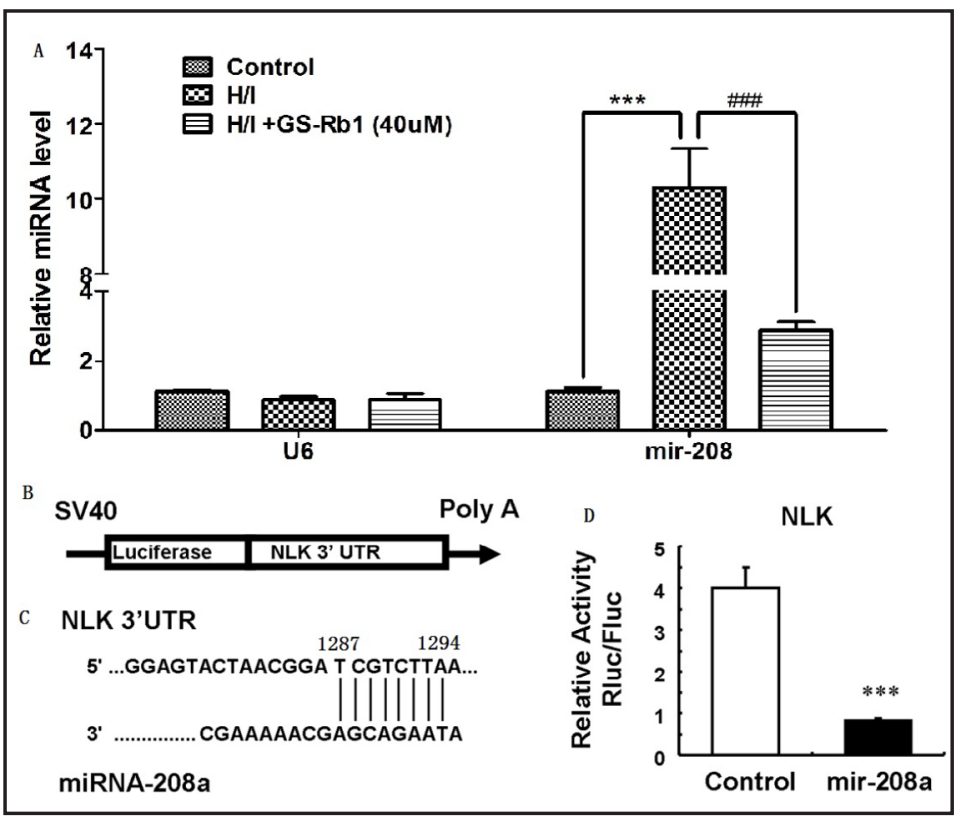

Fig. 3. NLK protein expression was inhibited by the upregulation of miR-208 in NRCMs cells. (A) The real time PCR shows that the NLK gene was suppressed by the over expression of mir-208. (B) A representation of western blotting showing the expression of NLK protein was suppressed by the upregulation of miR-208. $* * * \mathrm{P}<0.001$ versus negative control group. This indicates that miR-208 inhibited NLK protein expression in NRCMs cells.

Fig. 4. Impact of miR-208 on the cardioprotective effect of Rb1. (A) NRCMs was transfected with miR-208 blank, mimics and inhibitors. Phase contrast light microscope fluorescence images of the cells were taken At $48 \mathrm{~h}$ after transfection. (B) MTT assay was performed to evaluated cell viability of each group.
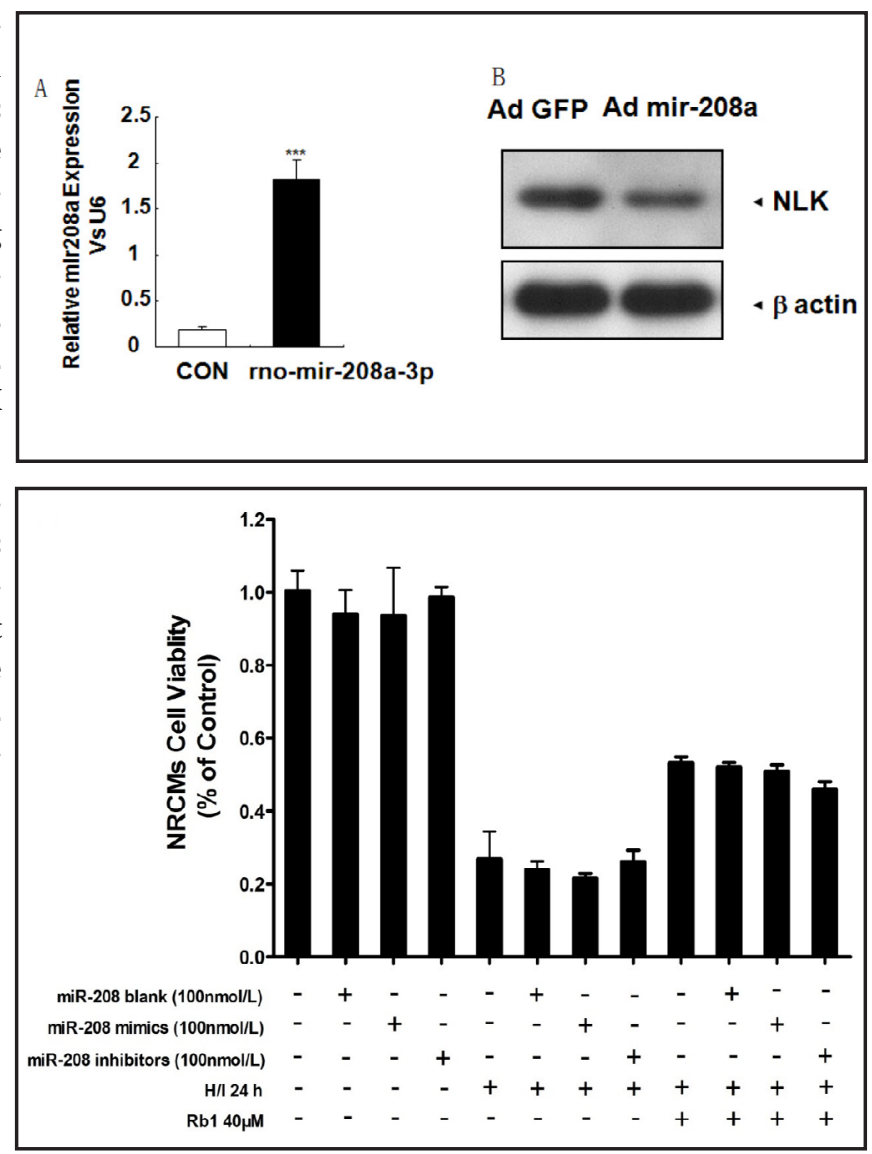

Impact of miR-208 on the cardioprotective effect of Rb1

In order to verify the impact of miR-208 on the cardioprotective effect of Rb1, miR208 blank, mimics and inhibitors were transfected into NRCMs (Fig. 4A). All the cells with or without $\mathrm{Rb} 1$ were subjected to $24 \mathrm{~h} \mathrm{H} / \mathrm{I}$ treatment. Cell viability was calculated by MTT assay. MiR-208 blank, mimics and inhibitors had no effect on the NRCMs viability, as well as response to the H/I injuries (Fig. 4B). But miR-208 inhibitor slightly decreased the protective 
Fig. 5. Impact of NLK on the cardioprotective effect of $\mathrm{Rb} 1$. (A) A representation and analysis data of western blotting showing the lentivirus-mediated overexpression of NLK in NRCMs with or without H/I injury. (B) MTT assay was performed to evaluated cell viability of each group. ${ }^{*} \mathrm{P}<0.05$ versus control group; ${ }^{~} \mathrm{P}<0.05$ versus $\mathrm{H} / \mathrm{I}$ group.

effect of Rb1 on the H/I impaired NRCMs. However, results showed no statistical difference.

Impact of NLK on the cardioprotective effect of $R b 1$

Since overexpression of miR-208 had no effect on the NRCMs viability, and given that NLK is a direct target of miR-208, we hypothesized that NLK may neither be involved in the cardioprotective effect of Rb1. To investigate this, we assessed the effect of NLK overexpression on NRCMs cell growth by MTT assay. Treatment of NRCMs with H/I injury suppressed the expression of NLK (Fig. $5 \mathrm{~A}$ ), while GS-Rb1 treatment significantly preserved the expression of NLK. Further more, the exogenous overexpression of NLK did not affect the NRCMs viability with or

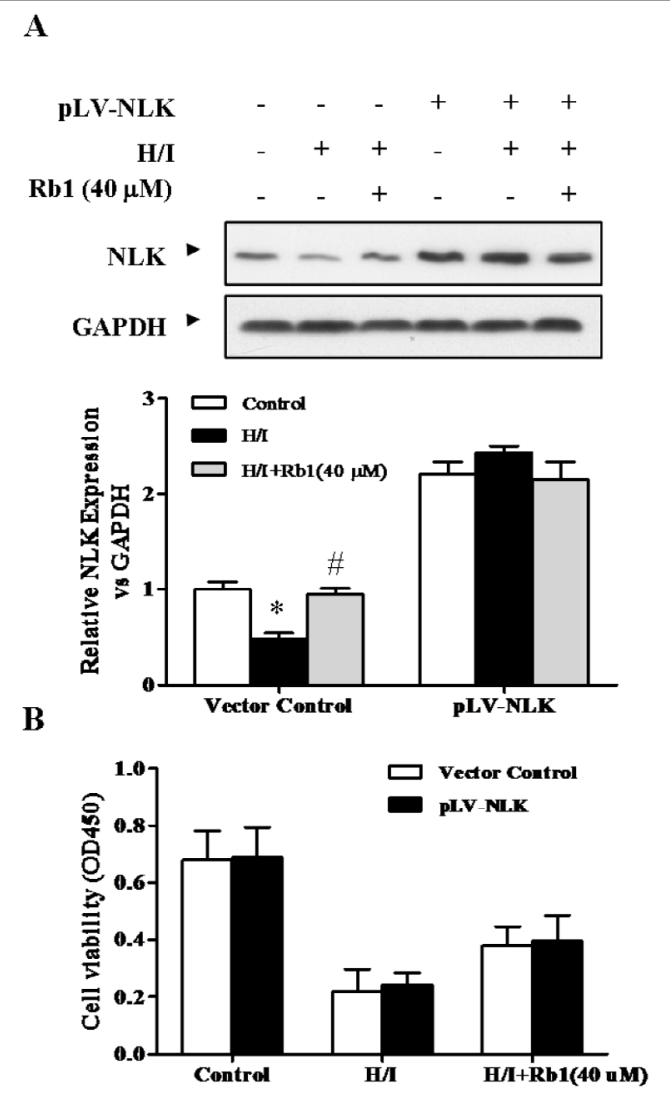
with not H/R injury. (Fig. 5B). This suggests that downregulation of NLK expression by miR208 did not involved in the cardioprotective effect of Rb1 against H/I injury.

\section{Discussion}

Mir-208, strictly expressed in cardiomyocytes, act as a biomarker for MI diagnosis. Therapeutic inhibition of miR-208a by subcutaneous treatment with anti-miR-208a during hypertension-induced heart failure in Dahl hypertensive rats prevented pathological myosin switching and cardiac remodeling, while improving cardiac functioning, overall health and survival [30]. With other target genes of mir-208, it has been proved that miR-208 was necessary for the production of alpha-myosin heavy chain and controlling the response of the heart to hypertrophic stimuli [20]. MiR-208 was also involved in insulin-induced vascular smooth muscle cell proliferation by downregulating p21, a key member of CDK-inhibitory protein family [31]. In preosteoblasts, miR-208 is closely involved in preosteoblast differentiation by partially regulating its target gene, V-ets erythroblastosis virus E26 oncogene homolog 1 (Ets1) [32]. However, we provide new that NLK was the target gene of mir-208 with regard to cardiomyocyte apoptosis.

The NLK gene could be related to diabetic hearts, like the mir-208 putative target gene [33]. This prediction of the NLK gene involvement was examined using TargetScan. human6.2. Western blotting was also showed that NLK was downregulated in the H/I group, whereas mir-208 was dramatically increased. Furthermore, to identify whether miR-208 can genuinely regulate the expression of NLK, NLK 3'-UTR was cloned into the pSiCHECK2, giving the 3'-UTR the majority of potential miRNA binding sites downstream of the coding sequence for luciferase (pMiR-Report; Promega). Cardiomyocytes were cotransfected with the pSiCHECK2 vector containing the 3'-UTR and miR-208 or negative miRNA. Overexpression of miR-208 significantly reduced luciferase activity from the reporter construct containing 


\section{Cellular Physiology Cell Physiol Biochem 2016;39:1187-1195

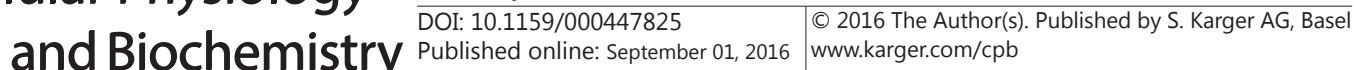 \\ Yan et al.: miR-208 Targets Nemo-Like Kinase in Cardiomycytes}

the NLK 3'-UTR, suggesting that NLK is a direct miR-208 target. Consider the fact that exogenous overexpression of NLK did not affect the NRCMs viability with or with not H/R injury, down-regulation of NLK expression by miR-208 is not involved in the cardioprotective effect of Rb1 against $\mathrm{H} / \mathrm{I}$ injury.

However, we only get the negative results when transfected miR-208 mimics and inhibitors into the NRSMs to prove the impact of miR-208 on cell viability and the cardioprotective effect of Rb1. We speculated that, miR-208 act indirectly during $\mathrm{Rb} 1$ protecting $\mathrm{H} / \mathrm{I}$ impaired NRCMs. We have proved that, several microRNAs involved in cardioprective properties of $\mathrm{Rb} 1$ [34] and $\mathrm{H} / \mathrm{I}$ induced cardiomyocytes apoptosis could be regulated by the mitochondria apoptotic pathway [29]. This leaves open the possibility of exploring the relationship of mir-208 with the mitochondrial apoptotic pathway in H/I induced cardiomyocyte apoptosis.

\section{Acknowledgments}

This work was supported by Grants from the National Natural Science Foundation of China (nos. 81273890 and 81173367), National key basic research development program (973 Program) (2015CB554400) and Postdoctoral Science Foundation of Beijing (2013ZZ$57)$.

\section{Disclosure Statement}

The authors declare that they have no conflict of interests.

\section{Reference}

1 D'Alessandra Y, Devanna P, Limana F, Straino S, Di Carlo A, Brambilla PG, Rubino M, Carena MC, Spazzafumo L, De Simone M: Circulating microRNAs are new and sensitive biomarkers of myocardial infarction. Eur Heart J 2010;31:2765-2773.

2 Zampetaki A, Willeit P, Tilling L, Drozdov I, Prokopi M, Renard J-M, Mayr A, Weger S, Schett G, Shah A: Prospective study on circulating MicroRNAs and risk of myocardial infarction. J Am Coll Cardiol 2012;60:290-299.

3 Xu Y, Zhu W, Wang Z, Yuan W, Sun Y, Liu H, Du Z. Combinatorial microRNAs suppress hypoxia-induced cardiomyocytes apoptosis. Cell Physiol Biochem 2015;37:921-932.

4 Wang Y, Men M, Yang W, Zheng H, Xue S. MiR-31 Downregulation Protects Against Cardiac Ischemia/ Reperfusion Injury by Targeting Protein Kinase C Epsilon (PKC $)$ ) Directly. Cell Physiol Biochem 2015;36:179-190.

5 Ishitani T, Ishitani S: Nemo-like kinase, a multifaceted cell signaling regulator. Cell Signal 2013;25:190-197.

6 Dohn TE, Waxman JS: Distinct phases of Wnt/ $\beta$-catenin signaling direct cardiomyocyte formation in zebrafish. Dev Biol 2012;361:364-376.

7 Naito AT, Shiojima I, Akazawa H, Hidaka K, Morisaki T, Kikuchi A, Komuro I: Developmental stage-specific biphasic roles of Wnt/ $\beta$-catenin signaling in cardiomyogenesis and hematopoiesis. Proc Natl Acad Sci U S A 2006;103:19812-19817.

8 Aisagbonhi O, Rai M, Ryzhov S, Atria N, Feoktistov I, Hatzopoulos AK: Experimental myocardial infarction triggers canonical Wnt signaling and endothelial-to-mesenchymal transition. Dis Model Mech 2011;4:469483.

9 Dawson K, Aflaki M, Nattel S: Role of the Wnt-Frizzled system in cardiac pathophysiology: a rapidly developing, poorly understood area with enormous potential. J Physiol 2013;591:1409-1432.

10 Malekar P, Hagenmueller M, Anyanwu A, Buss S, Streit MR, Weiss CS, Wolf D, Riffel J, Bauer A, Katus HA: Wnt signaling is critical for maladaptive cardiac hypertrophy and accelerates myocardial remodeling. Hypertension 2010;55:939-945.

11 Xi X-H, Wang Y, Li J, Wang F-W, Tian G-H, Yin M-S, Mu Y-L, Chong Z-Z: Activation of Wnt/ $\beta$-catenin/GSK3 $\beta$ signaling during the development of diabetic cardiomyopathy. Cardiovasc Pathol 2015;24:179-186. 


\section{Cellular Physiology Cell Physiol Biochem 2016;39:1187-1195 \begin{tabular}{l|l|l|l|l|l}
\hline DOI: 10.1159/000447825 & $\begin{array}{l}\text { () 2016 The Author(s). Published by S. Karger AG, Basel } \\
\text { www.karger.com/cpb }\end{array}$
\end{tabular}}

Yan et al.: miR-208 Targets Nemo-Like Kinase in Cardiomycytes

12 Creemers EE, Tijsen AJ, Pinto YM: Circulating microRNAs novel biomarkers and extracellular communicators in cardiovascular disease? Circ Res 2012;110:483-495.

13 Du W, Pan Z, Chen X, Wang L, Zhang Y, Li S, Liang H, Xu C, Zhang Y, Wu Y, Shan H, Lu Y. By targeting Stat3 microRNA-17-5p promotes cardiomyocyte apoptosis in response to ischemia followed by reperfusion. Cell Physiol Biochem 2014;34:955-965.

14 Shukla LI, Chinnusamy V, Sunkar R: The role of microRNAs and other endogenous small RNAs in plant stress responses. Biochim Biophys Acta 2008;1779:743-748.

15 Zhao X, Wang K, Liao Y, Zeng Q Li Y, Hu F, Liu Y, Meng K, Qian C, Zhang Q, Guan H, Feng K, Zhou Y, Du Y, Chen Z. MicroRNA-101a inhibits cardiac fibrosis induced by hypoxia via targeting TGFßRI on cardiac fibroblasts. Cell Physiol Biochem 2015;35:213-226.

16 Ambros V: The functions of animal microRNAs. Nature 2004;431:350-355.

17 Callis TE, Deng Z, Chen J-F, Wang D-Z: Muscling through the microRNA world. Exp Biol Med 2008;233:131138.

18 Kloosterman WP, Plasterk RH: The diverse functions of microRNAs in animal development and disease. Dev Cell 2006;11:441-450.

19 Lewis BP, Burge CB, Bartel DP: Conserved seed pairing, often flanked by adenosines, indicates that thousands of human genes are microRNA targets. Cell 2005;120:15-20.

20 van Rooij E, Sutherland LB, Qi X, Richardson JA, Hill J, Olson EN: Control of stress-dependent cardiac growth and gene expression by a microRNA. Science 2007;316:575-579.

21 Corsten MF, Dennert R, Jochems S, Kuznetsova T, Devaux Y, Hofstra L, Wagner DR, Staessen JA, Heymans S, Schroen B: Circulating MicroRNA-208b and MicroRNA-499 reflect myocardial damage in cardiovascular disease. Circ Cardiovasc Genet 2010;3:499-506.

22 Fichtlscherer S, Zeiher AM, Dimmeler S: Circulating MicroRNAs biomarkers or mediators of cardiovascular diseases? Arterioscler Thromb Vasc Biol 2011;31:2383-2390.

23 Ji X, Takahashi R, Hiura Y, Hirokawa G, Fukushima Y, Iwai N: Plasma miR-208 as a biomarker of myocardial injury. Clin Chem 2009;55:1944-1949.

24 Cheng Y, Wang X, Yang J, Duan X, Yao Y, Shi X, Chen Z, Fan Z, Liu X, Qin S: A translational study of urine miRNAs in acute myocardial infarction. J Mol Cell Cardiol 2012;53:668-676.

25 Bao C, Wang Y, Min H, Zhang M, Du X, Han R, Liu X. Combination of ginsenoside Rg1 and bone marrow mesenchymal stem cell transplantation in the treatment of cerebral ischemia reperfusion injury in rats. Cell Physiol Biochem 2015;37:901-910.

26 Liu S, Chen M, Li P, Wu Y, Chang C, Qiu Y, Cao L, Liu Z, Jia C. Ginsenoside rh2 inhibits cancer stem-like cells in skin squamous cell carcinoma. Cell Physiol Biochem 2015;36:499-508.

27 Wang RX, He RL, Jiao HX, Dai M, Mu YP, Hu Y, Wu ZJ, Sham JS, Lin MJ. Ginsenoside Rb1 attenuates agonistinduced contractile response via inhibition of store-operated calcium entry in pulmonary arteries of normal and pulmonary hypertensive rats. Cell Physiol Biochem 2015;35:1467-1481.

28 Wang X-f, Liu X-j, Zhou Q-m, Du J, Zhang T-l, Lu Y-y, Su S-b: Ginsenoside rb1 reduces isoproterenol-induced cardiomyocytes apoptosis in vitro and in vivo. Evid Based Complement Alternat Med 2013;2013:454389.

29 Yan X, Tian J, Wu H, Liu Y, Ren J, Zheng S, Zhang C, Yang C, Li Y, Wang S: Ginsenoside rb1 protects neonatal rat cardiomyocytes from hypoxia/ischemia induced apoptosis and inhibits activation of the mitochondrial apoptotic pathway. Evid Based Complement Alternat Med 2014;2014: 149195.

30 Montgomery RL, Hullinger TG, Semus HM, Dickinson BA, Seto AG, Lynch JM, Stack C, Latimer PA, Olson EN, van Rooij E: Therapeutic inhibition of miR-208a improves cardiac function and survival during heart failure. Circulation 2011;124:1537-1547.

31 Zhang Y, Wang Y, Wang X, Zhang Y, Eisner GM, Asico LD, Jose PA, Zeng C: Insulin promotes vascular smooth muscle cell proliferation via microRNA-208-mediated downregulation of p21. J Hypertens 2011;29:15601568.

32 Diao X, Shen E, Wang X, Hu B: Differentially expressed microRNAs and their target genes in the hearts of streptozotocin-induced diabetic mice. Mol Med Rep 2011;4:633-640.

33 Bergmann MW: WNT Signaling in Adult Cardiac Hypertrophy and Remodeling Lessons Learned From Cardiac Development. Circ Res 2010;107:1198-1208.

34 Yan X, Xue J, Wu H, Wang S, Liu Y, Zheng S, Zhang C, Yang C: Ginsenoside-Rb1 Protects Hypoxic-and Ischemic-Damaged Cardiomyocytes by Regulating Expression of miRNAs. Evid Based Complement Alternat Med 2015;2015: 171306. 